\title{
SOBRE LA EDICIÓN DE REVISTAS DISCIPLINARES E INTERDISCIPLINARES EN EL CAMPO DE LAS CIENCIAS SOCIALES
}

\author{
Héctor VÁZQUEZ ${ }^{1}$ \\ Margot BIGOT
}

\section{Resumen}

En este trabajo exponemos la trayectoria de la Revista Papeles de Trabajo que se publico desde 1991 a 2007 impresa en papel ,y desde 2008 , en la plataforma on line de Scielo Argentina. Y nos referiremos, en la medida de nuestras posibilidades, a la situación editorial de las revistas científicas de ciencias sociales que se publican en La Argentina. Palabras clave: Revistas de ciencias sociales- medios electrónicos,-acceso abierto

\begin{abstract}
In this paper the authors refer to the trajectory of the Journal review Papeles de Trabajo, that has been published since 1991 to 2007 in printed form, and since 2008 in the on line platform of Scielo Argentina. They also refer, as far as they find it possible, to the editorial situation of social sciences journals published in Argentina.
\end{abstract}

Key Words: social sciences journals- elctronic media- open acces

\section{Resume}

\footnotetext{
${ }^{1}$ Editores de la revista Papeles de Trabajo. Plataforma SciELO Argentina. Centro de Estudios Interdisciplinarios en Ciencias Etnolingüísticas y Antropológico-sociales. Facultad de Humanidades y Artes UNR.
} 
Dans cet article, nous présentons la trajectoire de la revue Papeles de Trabajo, publié de 1991 à 2007 imprimé sur papier et, depuis 2008, sur la plateforme en ligne de SciELO Argentine. Et nous ferons référence, dans la mesure de nos possibilités, à la situation éditoriale des revues scientifiques de sciences sociales publiées en Argentine.

Mots-clés: Revues en sciences sociales - Médias électroniques, - Accès ouvert

\section{Proyecto Editorial}

La revista "Papeles de Trabajo" (ISSN 1852-450), es editada por el Centro Interdisciplinario de Ciencias Etnolingüísticas y Antropológico-Sociales (CICEA), uno de los Centros de Investigación de la Facultad de Humanidades y Artes de la Universidad Nacional de Rosario,

Desde 1991 a 2007 el CICEA editó, con periodicidad anual, la Revista "Papeles de Trabajo" (ISSN 0327-6147) impresa en papel. A partir del 2007 se publicó en la plataforma on line de SciELO Argentina, lo que nos permitió acceder al universo internacional de revistas científicas $\mathrm{y}$, por lo tanto, a un nivel cualitativamente muy superior. La presencia de la revista en la "comunidad científica" internacional nos permite un nivel de impacto que no teníamos y habilita el intercambio de opiniones entre cientistas sociales enriqueciendo la problemática tratada.

El equipo de trabajo consta de:

- dos directores y editores: Héctor VÁZQUEZ y Margot BIGOT

- un Consejo Editorial integrado por investigadores de:

La Universidad de Buenos Aires,

Universidad Nacional de la Plata,

Universidad Nacional Autónoma de México (UNAM), 
Centro de Investigaciones y Estudios Superiores de Antropología Social. (CIESAS). México

Universidad Federal de Minas Gerais - Brasil

École des Hautes Études en Sciences Sociales (EHESS) de Paris

Del CONICET y del Consejo de Investigaciones de la Universidad Nacional de Rosario CIUNR

La impresión de la Revista se realizaba en la imprenta del CONICET-ROSARIO y para bajar los costos teníamos que entregar los originales diseñados y listos para imprimir, además de proporcionar los materiales necesarios para la impresión. Todo esto insumía muchas horas de nuestro trabajo.

La revista se financiaba por: recursos propios del CICEA y la venta de ejemplares, cuya distribución era dificultosa

La Asociación Cooperadora “José Pedroni” de la Facultad de Humanidades y Artes- UNR siempre colaboró (y lo sigue haciendo actualmente)

$\mathrm{Y}$, algunos números, se financiaron con dinero otorgado por la Agencia Nacional de Promoción Científica y Tecnológica (PICT nº 04-9951 y por CONICET (PID Nº 03036.

En 2004 cumpliendo con los parámetros de calidad editorial del Sistema Latindex (Sistema Regional de Información en Línea para Revistas Científicas de América Latina, el Caribe, España y Portugal) fue evaluada en el Nivel Uno, e incluida en el Catálogo de Latindex

En 2007, se cumplieron los requisitos 1 para ingresar en el Núcleo Básico de Revistas Científicas Argentinas, fue evaluada por el Comité Científico Asesor (integrado por investigadores, editores, docentes universitarios de reconocido prestigio que representan equitativamente las distintas áreas del conocimiento) designado por el Directorio del CONICET. Y, por Resolución 1071/07 del CONICET, la revista PAPELES DE TRABAJO fue incorporada al Núcleo Básico de Revistas Científicas Argentinas, 
El Núcleo Básico de Revistas está conformado por el conjunto de las publicaciones científicas y tecnológicas editadas en el país que poseen mayor calidad editorial y de contenidos, que cuentan con mecanismos de evaluación

Acorde con criterios internacionales, y con el reconocimiento de la comunidad científica de su área.

La inclusión de Papeles de Trabajo en el Núcleo Básico ofreció la posibilidad de incorporarla al portal SciELO- Argentina, (Scientific Electronic Library Online) que es una biblioteca electrónica que conforma una red iberoamericana de colecciones de revistas científicas de excelencia, con texto completo, en soporte electrónico y con acceso abierto, libre y gratuito. Cuyo fin es otorgar visibilidad a la producción científica nacional y regional (fue inaugurado en Argentina en 2005).

NÚCLEO BÁSICO. Las revistas deberán cumplir con los siguientes requisitos, según consta en la Resolución N 1640 del CONICET. a) Contar con un comité editorial integrado por pares especialistas en su temática. b) Estar indizadas en bases de datos internacionales. c) Contener un alto porcentaje de artículos y notas breves originales. d) Publicar mayoritariamente materiales de autores externos a la entidad editora. e) Someter los artículos publicados a estricto arbitraje externo. f) Ser reconocidas por su trayectoria y liderazgo en su temática y contar con respaldo institucional, académico o profesional. g) Ser editadas regularmente, de acuerdo con la periodicidad declarada por sus editores. h) Respetar, en su diseño y formato, las normas editoriales internacionales, cumpliendo con los parámetros de calidad editorial del Sistema Latindex (Sistema Regional de Información en Línea para Revistas Científicas de América Latina, el Caribe, España y Portugal) (haber evaluado en el Nivel I y estar incluidas en el Catálogo de Latindex). i) Estar inscriptas en el Centro Nacional Argentino del ISSN -Número Internacional Normalizado de Publicaciones Seriadas (International Standard Serial Number) El sistema ISSN es un programa de la UNESCO/UNISIST (sistema mundial de información)- del CAICYT (Centro Argentino de Información Científica y Tecnológica) y haber publicado este código. j) Cumplir con las normas internacionales en materia de conflicto de intereses y normas éticas. 
Desde 2007 ( N $^{\circ}$ 15) se publica en SciELO- Argentina, (Scientific Electronic Library Online). Fue reevaluada cada tres años, ratificada su permanencia en Latindex, y acreditada su publicación en Scielo hasta la actualidad, la última reevaluación fue este año

La facilidad de preparación del material (en Word y PDF), del envío, y el bajo costo han permitido que se publique con periodicidad semestral.

Actualmente estamos preparando EL No 34 que estará en ScIELO en Diciembre 2017.

Los autores son consultados y citados. Los artículos de la revista han sido visitados por lectores de numerosos países (Argentina, China, EEUU. Ucrania, México, Colombia, Rusia, España, Austria, Japón., etc.)

La gran difusión de los artículos publicados en ScIELO ha aumentado también muchísimo la cantidad de trabajos que recibimos para evaluar

Publica trabajos originales y artículos científicos actualizados que tratan aspectos teóricos y empíricos, a nivel disciplinario, multidisciplinario e interdisciplinario, en dominios de la Antropología Sociocultural (Antropología Lingüística, Antropología Jurídica, Antropología Médica) y Ciencias Sociales en general.

En esta ocasión hablaremos desde la perspectiva de editores de esta revista, y luego desde allí, nos referiremos, en la medida de nuestras posibilidades, a la situación editorial de las revistas científicas de ciencias sociales que se publican en La Argentina.

Hay claridad cuando nos referimos a la publicación de trabajo científicos disciplinares. Todos sabemos de qué se trata. Sin embargo conviene aclarar que el concepto de disciplina refiere a la enseñanza de uno o varios aspectos de la realidad (Heim Heckhausen 1975). En el caso de las ciencias sociales se trata de los criterios mediante cuales se construyen enfoques teóricos que procuran comprender la naturaleza y el comportamiento de un grupo delimitado de aspectos psico-socio-culturales metodológicamente articulados.

Por multidisciplinariedad entendemos la yuxtaposición de enfoques metodológicos diferentes sobre los mismos aspectos de la realidad (construidos como problemática de 
investigación) a partir desde distintas disciplinas sin llegar a establecer contactos entre sus zonas fronterizas. No hay convergencia interdisciplinaria.

Interdisciplinariedad: Se trata de una categoría solamente aplicable al proceso de investigación Supone una convergencia en núcleos interdisciplinares de distintas disciplinas sobre problemas de investigación. Implica, además, diferentes niveles de integración teórico-metodológicos entre cada disciplina mediante la elaboración de un común denominador (núcleo de convergencia interdisciplinar) a partir de los dilemas planteados en las zonas fronterizas intermedias. Esto supone un esfuerzo de síntesis. (Leo Apostel 1975).

¿De qué hablamos cuando hablamos de interdisciplinariedad? El problema del entrecruzamiento de conocimientos en función de la construcción de una temática de investigación no es de fácil resolución. La solución pasa 'por la reflexión conjunta de los investigadores en un trabajo de reflexión colectiva.

"La interdisciplinariedad", escribe Guy Michaud (1975) "no se aprende ni se enseña, se vive", y agrega "la interdisciplinariedad es el fruto de una formación continua, de una flexibilización de las estructuras mentales".

Por lo tanto, el trabajo en equipo plantea la tarea de investigación como labor colectiva. Se necesita formar equipos divididos en subequipos, cada uno de los cuales debe ser coordinado por un investigador. Habrá un coordinador general que intercoordinará los distintos subequipos. Sin embargo, y a pesar de la división del trabajo en equipos- y dentro del mismo equipo- cada miembro se interesará por relacionar los aspectos específicos de su trabajo con los del conjunto de la investigación.

Por lo demás, el coordinador de cada equipo, además de funcionar como mediador entre los aspectos particulares y el conjunto de la investigación, conectará los logros parciales de su equipo con los plasmados en los otros. Con tal propósito se realizarán reuniones periódicas entre ellos. Esto es fundamental en la investigación interdisciplinar pues permite una concepción más amplia, y por lo tanto más abarcativa y crítica, del campo de inflexión utilizado (el modo en los que los investigadores se insertan en el proceso de investigación) y de los dilemas y soluciones encontrados por los investigadores. 
A modo de ejemplo, y sin pretender ponernos como modelo, diremos que en una investigación interdisciplinar que realizamos dentro del contexto de las relaciones interétnicas sobre los asentamientos de los aborígenes tobas en la ciudad de Rosario (provincia de Santa Fe) y grupos de aborígenes residentes en zonas chaqueñas del Centro Chaco occidental implementamos en el campo de inflexión planteado en el proyecto:

1. Una aproximación histórico antropológica: análisis y diagnóstico de la realidad histórica y sociocultural de los grupos sociales a estudiar en sus interrelaciones con los distintos segmentos de la sociedad regional / nacional con los que interactúan, y de los simbolismos y procesos de síncresis religiosa. Este equipo se integró con historiadores, entnohistoriadores y antropólogos socioculturales.

2. Una aproximación etnolingüística: que investiga la estructura de la lenguaestructura social-sistema lógico clasificatorio, con el propósito de poner en evidencia la estructura gramatosemántica de la lengua qom y sus esquemas psicolingüísticos. Este subequipo se integra con etnolingüístas y antropólogos socioculturales.

3. Una aproximación socio-económica: trabaja sobre las modalidades de producción y de reproducción económico-social dentro del contexto de una formación histórico social determinada. . Este subquipo se encuentra integrado por ecólogos, etnobotánicos y antropólogos socioculturales.

4. Una aproximación socio-médica: Se interesa por confeccionar diagnósticos socio- médicos. Creencias .Creencias y comportamientos sobre el proceso de salud/ enfermedad/ atención y la etnomedicina. Está integrado por antropólogos socioculturales, especialistas en cuestiones de salud y agentes sanitarios.

Estas aproximaciones que dentro de un dominio de alcance medio se implican y autoimplican, suponen la convergencia- establecida en distintos planos y niveles- de núcleos interdisciplinares. Tales núcleos no son estáticos sino que se reelaboran constantemente según la dinámica impuesta por el proceso de investigación.

La construcción de nódulos de convergencia sobre un referente empírico determinado es labor de los investigadores. Ellos deben debatir las modalidades más provechosas de entrecruzamiento para resolver los problemas planteados. Esto exige un esfuerzo de 
creatividad capaz de abrir las posibilidades de construcción de nuevas categorías conceptuales, lo suficientemente flexibles para soportar- en varios dominios y niveleslas variaciones de sentido que deben explicitarse muy claramente. Todo abordaje interdisciplinario es, por definición, multidimensional, por lo que su estructura interna ha de adecuarse operativamente a esta exigencia.

Nos disculpamos por estas disquisiciones, pero resultan necesarias para comprender que estas características deben encontrarse en los trabajos que se pretenden interdisciplinares y se presentan para su evaluación para su posible publicación. Como insiste Margot Bigot, muchos de los trabajos que se pretenden interdisciplinares son multidisciplinares. Aunque sus autores no quieren aceptarlo. En ocasiones, se produce aquí un debate entre autores y editores en el que participan también los evaluadores.

La dificultad para conseguir evaluadores capacitados para juzgar estos trabajos es una segunda gran dificultad. El concepto de interdisciplinariedad se comprende vagamente y de un modo insuficiente. De ahí la dificultad de evaluar estos trabajos que confunden también a las Comisiones Asesoras de las instituciones de investigación que no saben cómo ubicarlos porque el criterio dominantes es la disciplinariedad. De todos modos, la presentación de trabajos interdisciplinares ha disminuido mucho. Últimamente sólo nuestro equipo interdisciplinar los presenta. Porque además de publicar en otras revistas científicas, de vez en cuando lo hacemos en PAPELES DE TRABAJO.

Perspectiva situada: El Proyecto Editorial se interesa especialmente en la publicación de trabajos cuyos contenidos intentan descentrarse de la epistemología de las ciencias sociales construida en los países centrales, a través de un pensamiento crítico y emancipador. Ubicándose, a partir del proceso de investigación situado aquí y ahora, en una perspectiva periférica, en una Antropología del Sur, capaz de reflexionar por fuera de las elaboraciones teóricas de la "civilización noratlántica" hegemónica y dominante, y priorizar, así, los trabajos

construidos con base a la realidad histórico-socio-cultural latinoamericana sobre la que se reflexiona críticamente, y en evidente disputa con los modos dominantes de la escritura antropológica de nuestros días.

Dicho esto, resulta muy importante aclarar que se evalúan todos los trabajos que recibe la revista, sin excepción alguna. De tal manera se procura seguir un criterio amplio y 
abarcativo carente de discriminaciones arbitrarias. De modo que el "Corte del Editor" está ausente, con excepción de trabajos presentados que en nada tengan que ver con las ciencias sociales; lo que resulta muy infrecuente.

Siguiendo las derivaciones de tal política editorial publicamos trabajos solamente en español, aunque no tendríamos problemas en hacerlo también en portugués. Debemos aclarar que no recibimos hasta el momento trabajos en esta lengua. Y las respectivas palabras claves en español, francés e inglés con el propósito de ampliar la difusión de los contenidos del texto publicado.

Otro de los dilemas que surgen durante el proceso de evaluación de los trabajos presentados, es el de la dificultad de conseguir evaluadores radicados en instituciones académicas y de investigación de los países centrales los que, en muchas ocasiones, son pocos receptivos a la propuesta y a veces, cuando la aceptan, no acuerdan con las líneas de abordajes de los textos y se muestran remisos a aceptarlos si no se entroncan en la línea teórica que ellos cultivan.

En ocasiones la relación entre los editores y el Consejo Editorial nos son suficientemente fluidas, en parte por nuestra responsabilidad.

La modalidad de arbitraje implementada es la del arbitraje ciego. En la mayoría de los casos la respuesta de los árbitros se demora demasiado. Aunque se le impone el plazo de un mes para la devolución del trabajo ya evaluado (quince días nos parece irreal ya que se trata de un plazo demasiado exiguo) la devolución se demora. El plazo se incumple y resulta necesario insistir, en ocasiones demasiado.

En tanto editores estamos conformes con la calidad de los trabajos publicados, sus aportes a las problemáticas tratadas y el estilo de redacción presentado.

\section{Acerca de las revistas argentinas de ciencias sociales.}

Desde una visión panorámica se puede argumentar que estas revistas en, La Argentina, son susceptibles de ubicarse en dos niveles considerando los criterios de visibilidad e internalización. Estos niveles son entonces: 
Nivel 1.- Revistas publicadas en plataformas internaciones ScIELO que forman parte del Núcleo Básico del CAYCIT.

Nivel 2.- revistas indexadas pero que no alcanzaron una plataforma internacional de difusión.

Transcribo una interesante cita sobre el tema:

"El problema de la visibilidad en revistas científicas de HyCS Página 10 que se afianzó en 2004 con el SCImago Journal Rank Indicator de Scopus/Scimago 16, restringieron el concepto de visibilidad en dos sentidos, desde la metodología utilizada y desde la escasa inclusión en ambas bases de datos de revistas no anglosajonas, particularmente es nuestro caso las de ALyC (AméricaLlatina y el Caribe) y especialmente en las HyCS (Historia y Ciencias Sociales) Ambos sistemas internacionales intentan reemplazar el trabajo de los colegas en el colegio invisible indicando, con análisis cuantitativos basados en conjuntos de revistas con poca representación de nuestra región, y en análisis de citaciones en esos conjuntos exclusivos de revistas, qué revistas son nucleares y prestigiosas en cada disciplina, formando un colegio invisible internacional que pretende definir la ciencia central. A los dos elementos que intervienen en los conceptos de calidad y visibilidad de las revistas científicas, colegio invisible y análisis de citación, se le suma, en la actualidad, un tercer elemento el acceso en línea a los contenidos completos de las revistas vía web, que se fortalece con el surgimiento del Movimiento de Acceso Abierto17. En la región los dos sistemas que han impulsado el acceso abierto a la información científica son SciELO y RedALyC con más de 1.000 títulos de revistas disponibles a texto completo sin restricciones. Existe un conjunto significativo de revistas que no están incluidas en los sistemas internacionales o regionales hasta ahora mencionados, ni utilizan la Internet para difundir sus contenidos, pero mantienen su prestigio porque el colegio invisible sigue siendo el eje vertebrador del desarrollo de estas disciplinas y, consecuentemente, de las revistas científicas que las sustentan. Entonces, es necesario redefinir el concepto de visibilidad, para que la originalidad de las contribuciones y la evaluación por pares sean los elementos principales que dan calidad y prestigio a una revista científica, siendo la visibilidad una variable de difusión, accesibilidad y ampliación del colegio invisible. Es por ello que urge la necesidad de identificar y brindar visibilidad, en el sentido de difundir 
su existencia y calidad científica, a todas aquellas revistas que contengan aportes originales de investigación y se comprometan a una rigurosa revisión por pares. El compromiso de conformar estos núcleos de revistas con prestigio disciplinar debería comenzar por cada país, para ofrecer un aval de calidad que brinde a los editores nacionales un elemento de peso a la hora de presentarse a los sistemas regionales o internacionales de indización" (Cecilia Rosemblum: 10)

El concepto de colegio invisible que utiliza Rosemblum refiere al de los pares evaluadores. La redefinición de la visibilidad que plantea disminuye el peso del concepto de impacto basado en el número de citaciones a niveles internacionales, siempre sesgado de acuerdo a intereses de conocimiento y problemáticas que interesan fuertemente a la producción científica de los países centrales, y pone de relieve la necesidad de considerar el valor científico de aquellas revistas que contengan artículos de contenido original y rigurosa evaluación por pares.

Las revistas de NIVEL 1.- Se encuentran en la lista alfabética de revistas científicas que presenta Scielo Argentina. Tales como Interdisciplinaria, revista de Psicología y ciencias sociales del Centro Interamericano de Investigaciones Psicológicas y ciencias afines (CIIPA) Universidad del Salvador-CONICET. $a V a$, revista de antropología. Secretaría de Investigación y Posgrado de la Facultad de Humanidades y Ciencias Sociales de la Universidad Nacional de Misiones. Runa de la Facultad de Filosofía y Letras UBA. Cuadernos de Antropología Social Facultad de Filosofía y Letras UBA. Cuadernos, Facultad de Humanidades y Ciencias Sociales. Universidad Nacional de Jujuy.

Este es el caso de las revistas científicas que para nosotros se ubican en el Nivel 2.Mencionemos solamente algunas: La Revista de la Escuela de Antropología, Facultad de Humanidades y Artes UNR (Latindex). CUADERNOS DEL INALP. Instituto Nacional de Antropología y Pensamiento Latinoamericano. Ciudad Autónoma de Buenos Aires. Publicar. Revista de Graduados en Antropología (REBID, Latindex y Doaj).Revista de Antropología (REBID) del Antropología del Museo de Ciencias Naturales y Antropológicas Antonio Serrano. Ministerio de Cultura y Educación de Provincia de Entre Ríos. Revista de Epistemología y Ciencias Humanas. Grupo IANUS, docentes de la Universidad Nacional de Rosario y de la Universidad Nacional del Litoral. 
Otras revistas: Publicadas por instituciones de dos países como La Revista interdisciplinaria de Estudios Sociales. Colectivo de Estudios e Investigaciones Sociales (CEISO), Bahía Blanca, Argentina en asociación con el grupo interdisciplinar de Estudos e Pesquisa sobre Capitais Transnacionais, Estado, Classe Dominantes e conflictividades en América Latinas e caribe (GIERTALC) con el aval institucional del Instituto Latinoamericano, Sociedad e Política (ILASEP) da Universidade Federal de Integraco Latino-Americana. (Latindex y CAPAE) Brasil.

Sin temor a equivocarnos podemos afirmar que la problemática de las revistas científicas de Ciencias Sociales en La Argentina son presupuesto, y las que hemos mencionado anteriormente al referirnos a la cuestiones que debemos resolver como editores de la Revista Papeles de Trabajo.

\section{Bibliografía:}

Apostel, Leo 1975 Interdisciplinariedad problemas de enseñanza e investigación en las universidades Apostel, Leo, Jean Piaget y otros. . ANNUIES. México.

Bigot, Margot 2016 "La eficacia de los medios electrónicos de acceso abierto en el proceso de comunicar la producción científica" Jornada "Experiencias de acceso abierto a la información científico académica en la Universidad Nacional de Rosario" 17 de noviembre de 2016.

Heckhausen, Heim 1975 Interdisciplinariedad problemas de enseñanza e investigación en las universidades Apostel, Leo, Jean Piaget y otros. ANNUIES. México.

Michaud, Guy 1975 Interdisciplinariedad problemas de enseñanza e investigación en las universidades Apostel, Leo, Jean Piaget y otros. ANNUIES .México

Rosemblaun. Cecilia 2014: El problema de la visibilidad en revistas científicas argentinas de Humanidades y Ciencias Sociales. Estudios de casos en Historia y Filosofía, página Web. En Repositorio Institucional de la Facultad de Humanidades y Ciencias de la Educación. Universidad Nacional de La Plata.

Vázquez, Héctor: 1994 La investigación socio-cultural. Crítica de la razón teórica y de la razón instrumental. Editorial Biblos. Argentina. 